\title{
Effects of the step structure on the yield, operating temperature, and the noise in step-edge Josephson junction rf-SQUID magnetometers and gradiometers
}

\author{
M. Fardmanesh *,1, J. Schubert, M. Banzet, W. Zander, Y. Zhang, H.J. Krause \\ Institut für Schicht- und Ionentechnik, Forschungszentrum Jülich (FZJ) GmbH, D-52425 Jülich, Germany
}

\begin{abstract}
Step-edge Josephson junction rf-SQUID magnetometers and gradiometers were made using YBCO films on La-

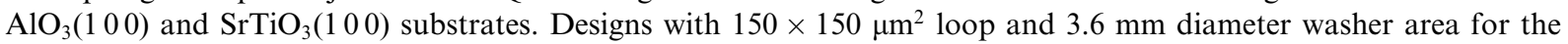
magnetometers $(230 \mathrm{pH})$, and $1.5 \mathrm{~mm}$ baseline and $1.5 \mathrm{~mm}$ diameter washer areas with a loop of $75 \times 75 \mu \mathrm{m}^{2}$ for the Gradiometers $(490 \mathrm{pH})$ were used. Effects of the step structure on the yield, optimal operating temperature range, and the $1 / f$ noise of the devices were investigated. The step structure was controlled using different ion beam etching (IBE) processes. The devices on $\mathrm{LaAlO}_{3}$ showed high sensitivity to the IBE parameters and the step structure while this was much less for the $\mathrm{SrTiO}_{3}$ substrate samples. This is mainly due to a considerable re-deposition of the substrate material on the step during the IBE process, in particular for $\mathrm{LaAlO}_{3}$, resulting in very low yield and high $1 / f$ noise devices. The film structure at the step was also found to be essentially dependent on the step structure strongly affecting the $1 / f$ noise of the devices. Using an optimized "combinational IBE" process, surface modified sharp steps were prepared resulting in high yield of low $1 / f$ noise devices when combined with high quality YBCO film. A typical $1 / f$ noise corner frequency of less than about $10 \mathrm{~Hz}$ with a white noise level of about $20 \mu \Phi_{\mathrm{o}} / \sqrt{\mathrm{Hz}}$ at liquid nitrogen temperature was obtained for these devices. The devices have shown stability over many thermal cycles and the time (over half a year since their fabrication) while kept at the room temperature environment. The operating temperature range of the devices was found to be controllable by the step depth and the film thickness for the steps. (C) 2001 Elsevier Science B.V. All rights reserved.
\end{abstract}

Keywords: rf-SQUID; Pulsed laser deposition YBCO; Step-edge Josephson junction; $1 / f$ Noise

\section{Introduction}

Single layer high- $T_{\mathrm{c}}$ step-edge Josephson junction (SEJ) rf-SQUIDs have superiority to other

\footnotetext{
${ }^{*}$ Corresponding author. Fax: +49-2461-612630.

E-mail address: m.fardmanesh@fz-juelich.de (M. Fardmanesh).

${ }^{1}$ On leave from Department of Electrical and Electronics Engineering, Bilkent University, Ankara, Turkey.
}

technology based SQUIDs due to the high flexibility in the design and ease in the application. However, many groups have observed a lack of reproducibility in the fabrication of stable SEJ rfSQUIDs, drastically reducing the yield of low $1 / f$ noise devices [1-3]. Producing sharp and clean steps has proved an essential first step in the fabrication technology of the SEJ SQUIDs [2-4]. Of course film quality and reproducibility is another hug parameter for obtaining high yield of low noise devices. 
In this work, the effects of the step structure on the noise and reproducibility of the rf-SQUID magnetometers and gradiometers as well as the film quality dependence are studied. In this paper we report on the development and implementation of ion beam etching (IBE) processes leading to high yield of low $1 / f$ noise YBCO SEJ rf-SQUIDs when combined with high quality films. The steps were prepared using different argon IBE parameters such as the beam angle and energy with different combinations of rotation of the substrate. Effects of the IBE process and the film quality on the $1 / f$ noise of the SQUIDs as well as on the operating temperature range and the amplitude of flux-voltage transfer function signal, $V_{\mathrm{s} \text {-pp }}$, are investigated. Similar step and film dependence behavior is observed for both magnetometers and gradiometers.

\section{Sample preparation and characterization setup}

The SQUID gradiometers and magnetometers were fabricated using $200 \mathrm{~nm}$ thick YBCO films on $\mathrm{LaAlO}_{3}\left(\begin{array}{lll}1 & 0 & 0\end{array}\right)$ and $\mathrm{SrTiO}_{3}\left(\begin{array}{lll}1 & 0 & 0\end{array}\right)$ substrates. The films were deposited using pulsed laser deposition technique using $\mathrm{KrF}_{2}$ excimer laser with $\lambda=248$ $\mathrm{nm}, 20 \mathrm{~ns}$ pulses, pulse energy of $1 \mathrm{~J}$ and up to 50 $\mathrm{Hz}$ repetition rate. The magnetometers were made with a design of $3 \mathrm{~mm}$ washer diameter and a loop of $150 \times 150 \mu \mathrm{m}^{2}(230 \mathrm{pH})$ [5]. The gradiometers were made with a design of $1.5 \mathrm{~mm}$ baseline and $75 \times 75 \mu \mathrm{m}^{2}$ loops $(490 \mathrm{pH})$. The films were patterned using conventional photolithography and low energy argon IBE as well as wet chemical etching. The steps were prepared using IBE with various beam angles and energies with different combinations of rotation of the substrates. Ion beam angles of $0-45^{\circ}$ from the normal incident to the substrate and ion beam energies from 250 to $500 \mathrm{eV}$ with $0.25-0.5 \mathrm{~mA} / \mathrm{cm}^{2}$ intensities were used. The devices were characterized by a temperature variable setup using our $1 \mathrm{GHz}$ rfSQUID electronics in $\mu$-metal shields allowing measurements down to about $5 \mathrm{~K}$ and a few $\mathrm{Hz}$.

\section{Dependence of the SQUID characteristics on the step structure}

The flux noise as well as the signal voltage, $V_{\text {s-pp }}$, of the SQUIDs versus temperature on different step structures were investigated. The $1 / f$ noise spectra of the SQUIDs were measured to be strongly dependent on the IBE process used for the step preparation. The optimum operating temperature of the SQUIDs was found to be strongly dependent on the step structure. This can be attributed to the fact that the height and the sharpness of the steps are affecting the $I_{\mathrm{c}}$ of the junctions. Therefore, a film thickness of $200 \mathrm{~nm}$ was used for all the devices studied in this work. The junctions of all our characterized low $1 / f$ noise devices show RSJ behavior with decrease of the normal resistance as the temperature increases. The detailed junction characteristics of the devices and their dependence on the step structure and the film will be presented elsewhere [5].

\subsection{Effects of ion beam etching process on the step structure and the $1 / f$ noise}

For the normal incident ion beam etched steps $\left(\theta=0^{\circ}\right)$, a hard layer of re-deposited substrate material is observed to form on the side walls of the steps with about $90 \mathrm{~nm}$ thickness and up to a few hundred nanometer height for $300 \mathrm{~nm}$ deep steps on $\mathrm{LaAlO}_{3}$ (Fig. 1, left). This effect was found to appear much less pronounced for the steps on $\mathrm{SrTiO}_{3}$ substrates. The re-deposited layer strongly reduces the yield of the SQUIDs to less than a few percent in particular for devices on $\mathrm{LaAlO}_{3}$. Also the SQUIDs made on such steps have shown high $1 / f$ noise, with a typical spectrum as in Fig. 2. The $1 / f$ noise of the devices on $\mathrm{SrTiO}_{3}$ was less sensitive to re-deposited material on the trench, leading to relatively higher yield of low $1 / f$ noise devices as compared to SQUIDs on $\mathrm{LaAlO}_{3}$.

For steps prepared using $45^{\circ}$ angled ion beam with substrate rotation, clean steps have been obtained for both type of the substrates. While preventing re-deposition of the materials, the steps prepared using $45^{\circ}$ angled beam with substrate rotation provide higher yield and lower $1 / f$ noise 


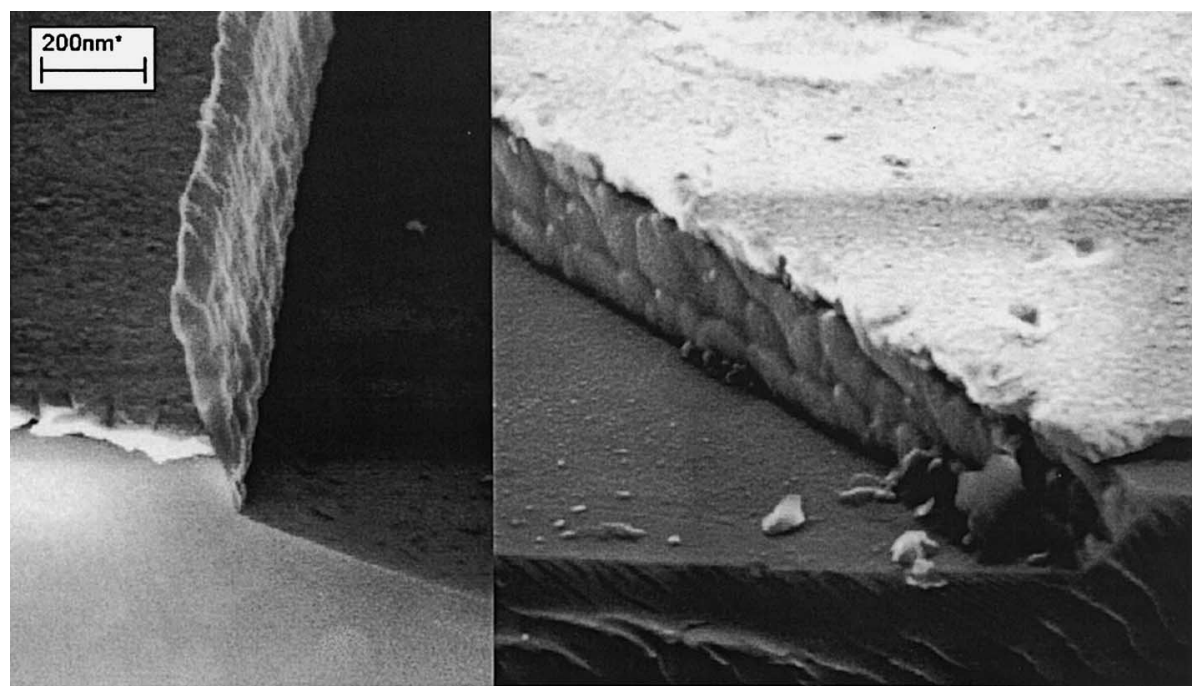

Fig. 1. SEM of $280 \mathrm{~nm}$ deep IB etched steps on $\mathrm{LaAlO}_{3}$ substrate made using non-optimized (left) and optimized (right) combinational IBE process.

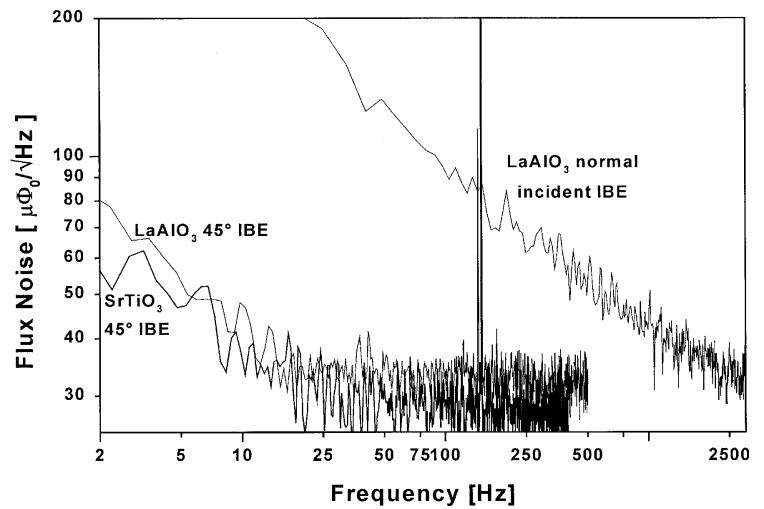

Fig. 2. Noise spectra of gradiometers made on $45^{\circ}$ and normal incident IB etched steps on $\mathrm{LaAlO}_{3}$ and $\mathrm{SrTiO}_{3}$ substrates.

SQUIDs as shown in Fig. 2, but with working temperatures higher than $77 \mathrm{~K}$. Due to the substrate rotation, angled ion beam creates curved structure at the trench, thus increasing the optimum operating temperature of the SQUIDs, $T_{\mathrm{Op}-\mathrm{j}}$, by increasing the $I_{\mathrm{c}}$ of the junction. The $T_{\mathrm{Op}-\mathrm{j}}$ could be lowered by increasing the depth of the steps. The curved region at the step also causes nonhomogeneous growth of the films with rough surfaces. The increase of the step depth led to further increase in the $1 / f$ noise of the devices.
This may be explained by further degradation of the film at the step.

An optimized combination of different angles and energy of incident ion beam process was developed to produce surface modified sharp steps shown in the Fig. 1 (right), while avoiding redeposition of the substrate material. This was done by conducting two etching steps. Using stationary angled ion beam along the trench with higher beam energy, the desired step depths were obtained. Then, rotating angled ion beam with lower beam energy was used to modify the surfaces of the steps. SQUIDs made on the sharp modified steps prepared by using the above combinational IBE process showed a high yield of low $1 / f$ noise devices. Typical noise spectra are shown in Fig. 3.

\subsection{Dependence of the optimum operating temper- ature of the SQUIDs on the step structure}

The dependence of the optimum operating temperature of the SQUIDs on the step structure was investigated using gradiometer array designs. The effect of the different junction widths on the $I_{\mathrm{c}}$ of the junctions and consequently on the $T_{\text {op-j }}$ was also investigated. The $T_{\text {op-j }}$ of gradiometers made of $200 \mathrm{~nm}$ thick YBCO film on a $\mathrm{LaAlO}_{3}$ substrate 


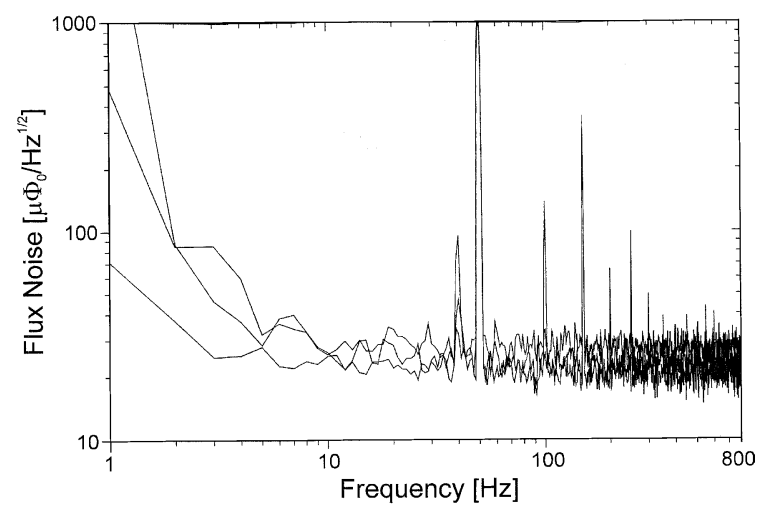

Fig. 3. Typical noise spectra at $77 \mathrm{~K}$ (liquid nitrogen temperature) of magnetometers on $\mathrm{LaAlO}_{3}$ substrate with $280 \mathrm{~nm}$ deep steps prepared using the combinational IBE method.

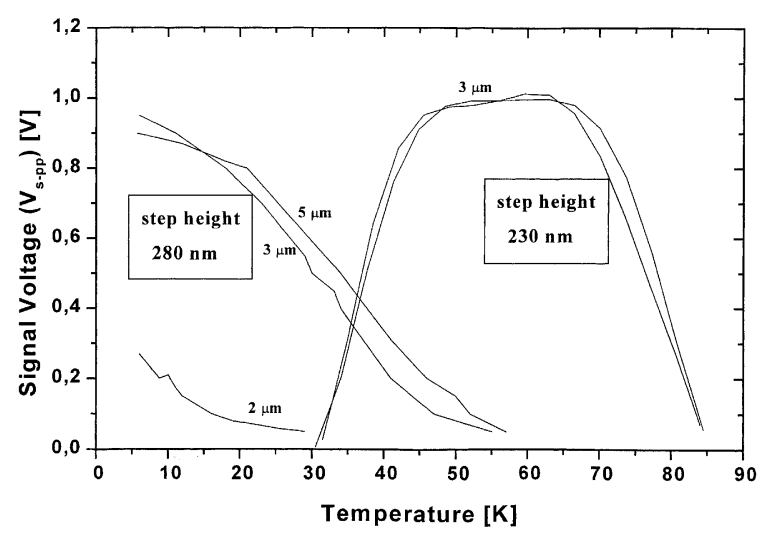

Fig. 4. $V_{\text {s-pp }}$ versus Temperature of 2, 3 and $5 \mu \mathrm{m}$ wide junction gradiometers on $\mathrm{LaAlO}_{3}$ with 230-280 nm deep steps prepared using the combinational IBE method.

with $280 \mathrm{~nm}$ deep steps was found to be below 30 $\mathrm{K}$, as shown in Fig. 4. While the operating temperature of junctions with 3 and $5 \mu \mathrm{m}$ width did not show a major difference, the operating temperature of $2 \mu \mathrm{m}$ wide junction was much lower as compared to the wider junctions. This effect is probably related to the effectiveness of precipitates in the films at the junction areas.

By lowering the step depth, the optimum operating temperature range of the SQUIDs could be raised to liquid nitrogen temperature. The film thickness of $200 \mathrm{~nm}$ was kept constant. The $V_{\mathrm{s} \text {-pp }}$ of SQUIDs with $3 \mu \mathrm{m}$ wide junction on a $\mathrm{LaAlO}_{3}$ substrate with $230 \mathrm{~nm}$ deep steps is shown in Fig.
4. The operating temperature range of the neighboring SQUIDs on one substrate were close to each other, spreading from about 40 to $85 \mathrm{~K}$. This is probably due to the high sensitivity of the critical current of the junctions on the structural properties and homogeneity of the YBCO film.

\section{The YBCO film and temperature dependence of the $1 / f$ noise spectra}

Noise spectra of SQUIDs show a correlation with the morphology of the films when measured versus temperature. Two types of temperature dependencies of $1 / f$ noise spectra were observed in the noisy devices. One which is associated with the precipitate density in the films, was found to increase with increase of the temperature. This is speculated to be due to increase of the thermal activation at higher temperatures for flux hopping process through precipitates in the films. High $1 / f$ noise spectra was measured for films with high concentration of precipitates as observed from the TEM and SEM graphs. The other type which is associated with the junction is measured to increase by decrease of temperature. This type of $1 / f$ noise spectra is for SQUIDs made of films with precipitates or imperfection only in the film areas very close to the junction, as well as for SQUIDs made on steps prepared using normal incident IBE. Temperature independent low $1 / f$ noise spectra shown in Fig. 3, was obtained for SQUIDs made of homogeneous low precipitate films both at the step edges and the washer areas on the steps prepared using the combinational IBE method.

\section{Summary and conclusions}

The effects of the step structure and the superconducting film on the yield and $1 / f$ noise of YBCO SEJ rf-SQUID magnetometers and gradiometers were investigated to obtain high yield of low noise devices. For the steps prepared using normal incident ion beam, a re-deposition, presumably of the substrate material, is observed to form on the side walls of the steps. The re-deposited material results in very low yield and high $1 / f$ 
noise devices. The re-deposition of the material and the sharpness of the steps could be controlled by an optimized combinational IBE method implementing variable beam parameters during the etching process. The temperature of the optimum performance of our SQUIDs, $T_{\text {op-j }}$, made on the modified sharp steps was controlled and tuned to $77 \mathrm{~K}$ by controlling the step height. The $T_{\text {op-j }}$ was decreasing by increase of the step height for a constant film thickness and junction width. Two types of $1 / f$ noise spectra were observed when measured versus temperature. One increasing with decrease of the temperature and was observed mostly in samples with re-deposited material at the step edge. It was also observed for devices made of films with precipitates and/or imperfection at the step. The other type increasing with increase of the temperature, was observed in devices made of films with high precipitate densities. Very high yield of low $1 / f$ noise SEJ rf-SQUIDs was obtained using high quality films on $\mathrm{LaAlO}_{3}$ substrates with steps prepared by the developed combinational IBE method.

\section{Acknowledgements}

We would like to thank A.I. Braginski and D. Koelle for their invaluable extensive discussions.

This work has been supported by the research and development project number 42.6.I3B.2.A.

\section{References}

[1] K. Herrmann, G. Kunkel, M. Siegel, J. Schubert, W. Zander, A.I. Braginski, C.L. Jia, B. Kabius, K. Urban, J. Appl. Phys. 7 (1995) 1131-1139.

[2] C.P. Foley, E.E. Mitchell, S.K.H. Lam, B. Sankrithyan, Y.M. Wilson, D.L. Tilbrook, S.J. Morris, IEEE Trans. Appl. Supercond. 9 (2) (1999) 4281-4284.

[3] A.I. Braginski, in: H. Weinstock (Ed.), SQUID Sensors: Fundamentals, Fabrication, and Applications, NATO ASI series, Kluwer, Dordrecht, 1996, p. 235.

[4] V.N. Glyantsev, M. Siegel, J. Schubert, W. Zander, A.I. Braginski, Supercond. Sci. Technol. 7 (1994) 253-255.

[5] M. Fardmanesh, J. Schubert, M. Banzet, W. Zander, Y. Zhang, Appl. Supercond. Conf. 2000, VA, USA, 17-22 September, 2000. 